Research Paper

\title{
FePt/GO Nanosheets Suppress Proliferation, Enhance Radiosensitization and Induce Autophagy of Human Non-Small Cell Lung Cancer Cells
}

Shijing $\mathrm{Ma}^{1 *}$, Hongtao Miao ${ }^{2 *}$, Yuan Luo ${ }^{1}$, Yingming Sun ${ }^{1}$, Xiaoli Tian ${ }^{1}$, Feng Wang ${ }^{1}$, Chengcheng You ${ }^{1}$, Shan Peng ${ }^{1}$, Guiliang Tang ${ }^{1}$, Cui Yang ${ }^{2}$, Wenjie Sun ${ }^{1}$, Shuying Li ${ }^{1}$, Yanling Mao' ${ }^{1}$, Jieyu Xu1 ${ }^{1}$, Yu Xiao ${ }^{3,4}$, Yan Gong $^{3}$, Hong Quan ${ }^{2}$, Conghua Xie ${ }^{1,5,6} \bowtie$

1. Department of Radiation and Medical Oncology, Zhongnan Hospital of Wuhan University, Wuhan, China

2. Key Laboratory of Artificial Micro- and Nano-Structures of Ministry of Education, School of Physics and Technology, Wuhan University, Wuhan, China

3. Department of Biological Repositories, Zhongnan Hospital of Wuhan University, Wuhan, China

4. Department of Urology, Zhongnan Hospital of Wuhan University, Wuhan, China

5. Hubei Key Laboratory of Tumour Biological Behaviors, Zhongnan Hospital of Wuhan University, Wuhan, China

6. Hubei Cancer Clinical Study Center, Zhongnan Hospital of Wuhan University, Wuhan, China

*These authors contributed equally to this work.

$\triangle$ Corresponding authors: Dr. Conghua Xie, Email: chxie_65@whu.edu.cn, Tel: +86-27-6781-2607, Fax: +86-27-6781-2892; and Dr. Hong Quan, Email: 00007962@whu.edu.cn, Tel: +86-13317101142, Fax: +86-27-68752569.

(C) Ivyspring International Publisher. This is an open access article distributed under the terms of the Creative Commons Attribution (CC BY-NC) license (https://creativecommons.org/licenses/by-nc/4.0/). See http://ivyspring.com/terms for full terms and conditions.

Received: 2018.09.09; Accepted: 2019.02.08; Published: 2019.03.10

\begin{abstract}
With the advancement of nanotechnology, various nanocomposites have been applied in the diagnostics and treatment of cancer. We synthetized FePt nanoparticles which were assembled on the surface of graphene oxide (GO). These novel FePt/GO nanosheets simultaneously act as a chemotherapy drug and enhance radiosensitivity. In this study, transmission electron microscope, dynamic light scattering, $X$-ray photoelectron spectroscope and Fourier transform infrared spectroscopy were used to characterize surface morphology and chemical composition of $\mathrm{FePt} / \mathrm{GO}$ nanosheets (NSs). Their cytotoxicity in various cancer and normal cells was evaluated by cell counting kit- 8 assay, and their effects on radiosensitization were determined by colony formation assay. To explore the underlying mechanisms, we measured the intracellular reactive oxygen species levels and autophagy formation. Monodansylcadaverine-staining, Western Blotting and ultrastructure analysis were utilized to assess autophagy. The results demonstrated that FePt/GO NSs not only selectively suppressed the proliferation of cancer cells, but also increased their radiosensitization. Moreover, $\mathrm{FePt} / \mathrm{GO}$ NSs induced autophagy, which might result in promoted sensibilization of radiotherapy. In conclusion, with good safety and efficacy, FePt/GO NSs are safe and effective to suppress proliferation, enhance radiosensitization and induce autophagy of human non-small cell lung cancer cells. They are potential for the treatment of lung cancer.
\end{abstract}

Key words: FePt/GO nanosheets, radiosensitization, lung cancer, autophagy

\section{Introduction}

Lung cancer is the most frequently diagnosed cancer and one of the leading causes of cancer death worldwide, among which non-small cell lung cancer (NSCLC) accounts for nearly $80 \%$ [1, 2]. Although traditionally well-established treatments (surgery, chemotherapy and radiotherapy) have made great contributions in saving patients' lives from lung cancer, the curable rate of lung cancer was still undesirable due to their own limitations. For instance, as the first-line treatment for NSCLC, cisplatin-based chemotherapy may lead to acquired drug resistance. Complications, such as radiodermatitis and radiation pneumonia, and tolerance dose of surrounding normal tissue limit the efficacy of radiotherapy. So, 
chemoradiotherapy, the concurrent administration of chemotherapy and radiotherapy, has been regarded as a significant paradigm in the treatment of NSCLC $[3,4]$. Although chemoradiotherapy improves local tumor control and curative rates, the agents used for chemoradiotherapy is still to be improved. The ideal agents should have desirable effects on both chemotherapy and radiotherapy without obvious toxicity in normal tissues.

The nanocomposites may be great agents due to their unique physicochemical characteristics in nanoscale. FePt nanocomposites were reported to have potential applications in dual modal MRI/CT imaging [5, 6], drug delivery [7], thermal therapy [8, 9] and chemotherapy agents [10]. FePt nanoparticles (NPs) inhibited the proliferation of cancer cells [11]. When combined with X-ray, they further inhibited the proliferation of HeLa cells due to their high X-ray absorption [12]. Therefore, FePt NPs could be suitable candidate for chemoradiotherapy agents.

Autophagy is a highly regulated catabolic process that degrades intracellular pathogens, damaged organelles and long-lived proteins as well as a cellular response to infection, starvation and disease progression [13]. Numerous human pathologies including neurodegenerative diseases, hereditary myopathies, abnormal immune responses and cancers were linked with autophagy, so the modulation of autophagy is potential to be an effective therapeutic strategy $[14,15]$. Previous researches suggested that intracellular nanoparticles induced autophagy and cytotoxicity [16-22]. Ma et al. [17] revealed that gold NPs induced autophagy accumulation by blocking autophagy flux. Yu et al. [21] demonstrated that silica NPs induced autophagy and autophagic cell death in HepG2 cells. Either autophagy overstimulation or blockage of autophagosome degradation induced by NPs could result in autophagy accumulation. Meanwhile, reactive oxygen species (ROS) played an important role in autophagy. ROS accumulation resulted in oxidative damage of proteins and mitochondria, which initiated autophagosome formation [23].

Graphene oxide (GO) is a favourite vector due to its flexible size, abundant oxygen-containing groups and excellent biocompatibility. To determine whether FePt/GO NSs could act as an agent in the chemoradiotherapy of NSCLC and to further explore the potential mechanisms, various NSCLC cells were used to assess their cytotoxicity in vitro. Viability assays, monodansylcadaverine-staining and ultrastructural analysis were used to examine their effects on radiosensitization, as well as autophagy. These results enriched our understanding on the cytotoxicity of NPs, which might be used in cancer management.

\section{Materials and Methods}

\section{Materials for FePt/GO NSs}

All chemicals and solvents were analytical grade. Chloroplatinic acid $\left(\mathrm{H}_{2} \mathrm{PtCl}_{6} \bullet 6 \mathrm{H}_{2} \mathrm{O}\right)$ was purchased from Reagent No. 1 Factory Of Shanghai Chemical Reagent. Iron acetylacetonate $\left(\mathrm{Fe}(\mathrm{acac})_{3}\right.$, $98 \%$ ) and graphite powder were purchased from Aladdin Reagent Co., Ltd. Oleylamine (OAm, > 70\%), oleic acid (OA) and sodium borohydride $\left(\mathrm{NaBH}_{4}\right.$, 96\%) were purchased from Sinopharm Chemical Reagent Co., Ltd.

\section{Synthesis of FePt/GO NSs}

GO was prepared from natural graphite powder by the modified Hummers method [24]. FePt NPs were obtained through a chemical reduction method. Briefly, $0.0682 \mathrm{~g} \mathrm{Fe}(\mathrm{acac})_{3}, 0.75 \mathrm{~mL}$ oleylamine and $0.75 \mathrm{~mL}$ oleic acid were added into $100 \mathrm{~mL}$ anhydrous ethanol and magnetism stirred at room temperature for $30 \mathrm{~min}$; then, $10 \mathrm{~mL} \mathrm{H}_{2} \mathrm{PtCl}_{6} \bullet 6 \mathrm{H}_{2} \mathrm{O}$ ethanol solution $(10 \mathrm{mg} / \mathrm{mL})$ was added. Subsequently, 0.247 $\mathrm{g} \mathrm{NaBH} 4$ was dissolved in $100 \mathrm{~mL}$ anhydrous ethanol, which was slowly added into the mixture with the temperature heated to $40^{\circ} \mathrm{C}$ for $1 \mathrm{~h}$. The product was obtained by centrifugation at $10000 \mathrm{r} / \mathrm{min}$ for $15 \mathrm{~min}$ and rinsed 3 times with ethanol to remove impurities; finally, the black product was dried by vacuum drying oven overnight at $40^{\circ} \mathrm{C}$.

Fifty $\mathrm{mg}$ FePt and $10 \mathrm{mg}$ GO were added into 50 $\mathrm{mL}$ ethanol and shaked at room temperature for $1 \mathrm{~h}$, followed by sonication for $4 \mathrm{~h}$. The precipitate was washed with ethanol 3 times and centrifuged at 10000 $\mathrm{r} / \mathrm{min}$ for $10 \mathrm{~min}$.

\section{Characterization techniques}

The morphology of $\mathrm{FePt} / \mathrm{GO}$ NSs was determined by EM2100FEF- $\Omega$ transmission electron microscope (TEM, JEOL, Tokyo, Japan) at $200 \mathrm{kV}$. The size distribution was measured by dynamic light scattering (DLS, Zatasizer Nano ZSP, Malvern, England). X-ray photoelectron spectroscope (XPS, ESCALAB250Xi, Thermo Fisher, USA) was used to determine the surface atomic composition. Fourier transform infrared spectroscopy (FTIR, Nicolet5700, Thermo Fisher, USA) was utilized to examine the existence of oxygen-containing groups on the surface of $\mathrm{FePt} / \mathrm{GO}$ NSs.

\section{Cell culture}

HeLa cell line (human cervical carcinoma cancer), H460 cell line (human large cell lung cancer), HELF cell line (human embryonic lung fibroblast), A549 cell line, H1975 cell line (human NSCLC) and Lewis lung carcinoma (LLC) cells were purchased 
from the Type Culture Collection of Chinese Academy of Science, Shanghai, China. All of these cell lines were cultured in RPMI media (HyClone, USA) supplemented with $10 \%$ fetal bovine serum (HyClone, USA), antibiotics penicillin $(100 \mathrm{U} / \mathrm{mL})$ and streptomycin $(100 \mathrm{mg} / \mathrm{mL})$ (HyClone, USA) in $37^{\circ} \mathrm{C} 5 \% \mathrm{CO}_{2}$ incubator (Sanyo, Japan).

\section{Cytotoxicity assay}

The cytotoxicity of FePt/GO NSs was detected by Cell Counting Kit-8 (CCK-8, Dojindo, Japan). Cells were seeded into 96-well plate (8000 cells in $100 \mu \mathrm{L}$ medium) and cultured for $24 \mathrm{~h}$. The cells were then treated with different concentrations of $\mathrm{FePt} / \mathrm{GO}$ with or without ROS scavenger $\mathrm{N}$-acetyl cysteine (NAC) at $37^{\circ} \mathrm{C}$ for $24 \mathrm{~h}$. CCK-8 regents $(10 \mu \mathrm{L} /$ well $)$ was added and incubated at $37^{\circ} \mathrm{C}$ for $2 \mathrm{~h}$. The absorbance at 450 nm was measured by Rayto-6000 system (Rayto, China). The relative cell viability (\%) was calculated as the ratio of absorbance of treated group to that of control group.

\section{Radiation}

After incubated with FePt/GO NSs (0, 2, 5, 10, $15,25 \mu \mathrm{g} / \mathrm{mL}$ ) at $37{ }^{\circ} \mathrm{C}$ for $2 \mathrm{~h}, \mathrm{H} 1975$ cells were irradiated by the small animal radiation research platform (SARRP, PXI X-RAD 225Cx, CT, USA) from a $204 \mathrm{kV}$ photon beam.

\section{Colony Formation Assay}

H1975 cells (200, 200, 400, 800, 1000, 2000 cells/ well) were seeded into 6-well plates. After $12 \mathrm{~h}$, FePt/GO NSs $(25 \mu \mathrm{g} / \mathrm{mL})$ were added to the medium. After incubation at $37{ }^{\circ} \mathrm{C}$ for another $12 \mathrm{~h}$, the cells were irradiated with $0,1,2,4,6$ and 8 gray (Gy). Two weeks later, the colonies were fixed with $4 \%$ paraformaldehyde and stained with crystal violet. A "multitarget-single hitting" model [25] was applied to fit the survival curve.

\section{Measurement of Reactive Oxygen Species (ROS)}

To measure intracellular ROS production, $\mathrm{H} 1975$ cells $\left(1 \times 10^{6}\right.$ cells/well) were seeded into 6 -well plates and incubated at $37{ }^{\circ} \mathrm{C}$ for $12 \mathrm{~h}$. FePt/GO NSs at different concentrations were added into medium and incubated at $37^{\circ} \mathrm{C}$ for $24 \mathrm{~h}$. The cells were collected in $1 \mathrm{~mL} 1640$ medium without FBS, and incubated with DCFH-DA probe $(10 \mu \mathrm{M})$ in the dark at $37^{\circ} \mathrm{C}$ for 30 min. The cells were then washed with serum-free RPMI-1640 3 times and the fluorescence signals were recorded by flow cytometry (BD FACS AriaIII, USA).

\section{Apoptosis assay}

The H1975 cells were placed in 6-well plates. Once cell adhered, FePt/GO NSs $(25 \mu \mathrm{g} / \mathrm{ml})$ were added to the medium. After incubation at $37^{\circ} \mathrm{C}$ for 12 $\mathrm{h}$, the plates were irradiated with $4 \mathrm{~Gy}$ by the small animal radiation research platform. The cells were harvested $24 \mathrm{~h}$ later, washed twice with cold phosphate buffer saline, and stained with FITCconjugated annexin $\mathrm{V}$ for $20 \mathrm{~min}$ and propidiumiodide for 5 min (BD Biosciences Ltd., USA) in the dark. The stained cells were assessed by flow cytometry, and analyzed by FlowJo vX.0.7 software.

\section{Monodansylcadaverine (MDC) staining}

H1975 cells $\left(5 \times 10^{5}\right.$ cells/well) were seeded into 12-well plates and cultured for $24 \mathrm{~h}$. Cells were incubated with FePt/GO NSs $(25 \mu \mathrm{g} / \mathrm{mL})$ at $37^{\circ} \mathrm{C}$ for $24 \mathrm{~h}$, and then irradiated with $4 \mathrm{~Gy}$. Two hours later, the cells were washed twice with wash buffer and stained with $0.05 \mathrm{mM}$ MDC in fresh 1640 medium at $37^{\circ} \mathrm{C}$ in the dark for $30 \mathrm{~min}$. Finally, the cells were washed twice with wash buffer and observed by inverted fluorescence microscope (Olympus BX51) with the excitation wavelength $350 \mathrm{~nm}$ and blocking wavelength $512 \mathrm{~nm}$.

\section{Western blotting assay}

The cells were lysed in RIPA lysis buffer containing protease inhibitor and phosphatase inhibitor (Sigma-Aldrich Ltd., USA) on ice for $30 \mathrm{~min}$, vortex shocking every $6 \mathrm{~min}$. The total protein concentration was measured by Bradford protein assay kit (Bio-Rad Ltd., USA). The proteins were separated with 10-15\% SDS-PAGE (Bio-Rad Ltd., USA) and transferred to polyvinylidene fluoride (PVDF) membrane (Millipore Ltd., USA). After blocking with tris buffered saline tween containing 5\% non-fat milk for $1 \mathrm{~h}$, the membranes were incubated with primary antibodies (GAPDH, LC3, p62 and Beclin1, Proteintech Group, Inc) at $4{ }^{\circ} \mathrm{C}$ overnight. The membranes were then incubated with corresponding secondary antibodies at room temperature for $2 \mathrm{~h}$ and the proteins were detected by enhanced chemiluminescence reagent (Bio-Rad Ltd., USA).

\section{Transmission electron microscopy (TEM)}

The cells were washed with PBS 3 times and then collected by cell scraper. After centrifuged at 2000 $\mathrm{r} / \mathrm{min}$ for $5 \mathrm{~min}$, the supernatants were discarded. The cell pellets were fixed with $2.5 \%$ glutaraldehyde at room temperature for $1 \mathrm{~h}$, and then post-fixed with $1 \%$ osmium tetroxide buffer for $1 \mathrm{~h}$. Subsequently, an ascending series of alcohol were performed for dehydration before embedding samples in araldite. Ultrathin sections were observed by TEM (HT7700, Hitachi, Japan, 100kV).

\section{Animals}

Female C57BL/6 mice at 6-8 weeks were purch- 
ased from Beijing Vital River Laboratory Animal Technology Co., Ltd. (Beijing, China), and housed in a specific pathogen-free environment. All the animal experiments were approved by the Institutional Animal Care and Use Committee of Wuhan University. LLC cells $\left(6 \times 10^{6}\right.$ cells/mouse) were implanted subcutaneously into the left lower limb. When the tumor volume reached $100 \mathrm{~mm}^{3}$, mice were randomly grouped as $\mathrm{FePt} / \mathrm{GO} \mathrm{NSs}$ (20 mg intratumor injection every 2 days for 1 week), irradiation (SARRP, PXI X-RAD 225Cx, CT, USA,20 Gy/2 f), FePt/GO NSs combined with irradiation and negative control. The body weight and tumor volume $(0.5 \times$ length $\times$ width $^{2}$ ) were measured every other day. The animals were sacrificed when reached the Mice Welfare Endpoint. The tumor samples were later fixed in $4 \%$ PFA and then embedded in paraffin for H\&E staining.

\section{Statistical analysis}

All the data were presented as means \pm standard deviation (SD). Statistical analysis was carried out by using Graphpad Prism 7. The significance between

A
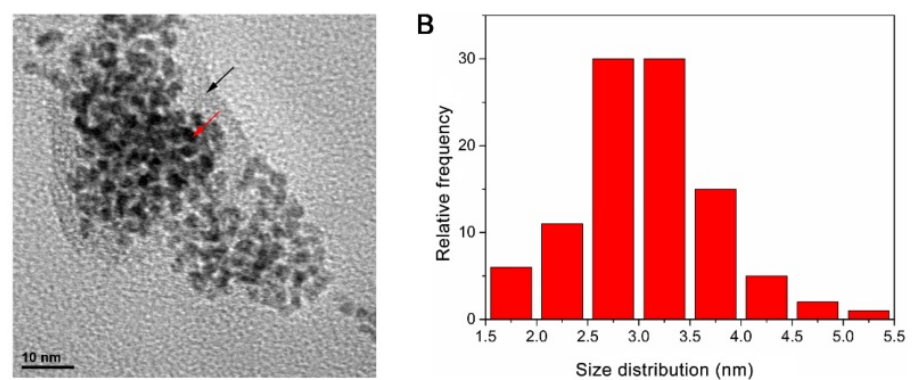

Size distribution $(\mathrm{nm})$
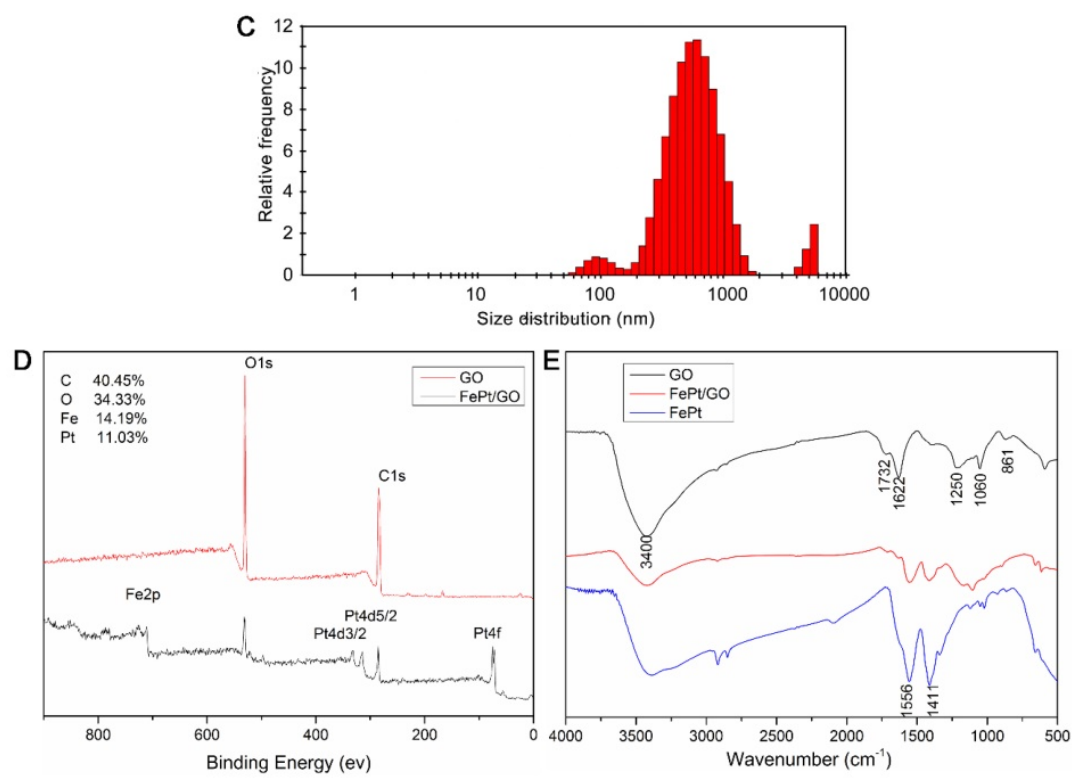

Figure 1. Characterization of FePt/GO NSs. (A) Representative TEM image of FePt/GO NSs. Black arrow indicates GO, and red arrow indicates FePt NPs. (B) The average diameter of FePt nanoparticles was $3.05 \mathrm{~nm}$. (C) The size distribution of FePt/GO NSs, whose mean diameter was $542.06 \mathrm{~nm}$. (D) The XPS spectrum of FePt/GO NSs, and the relatively atomic content of C, O, Fe, Pt were $40.45 \%, 34.33 \%, 14.19 \%$ and $11.03 \%$ respectively. (E) The FTIR spectrum of GO, FePt NPs and $\mathrm{FePt} / \mathrm{GO}$ NSs. different treatment groups was determined by one-way analysis of variance (ANOVA). Student's t-test was utilized to evaluate the differences of 2 groups. $\mathrm{P}<0.05$ was considered to be statistically significant.

\section{Results}

\section{Characterization of FePt/GO NSs}

The morphology and size distribution of FePt/GO NSs were determined by TEM and DLS. The spherical shape of FePt NPs were decorated on the GO surface (Fig. 1A) and the corresponding size distrubition (average diameter was $3.05 \mathrm{~nm}$ ) was calculated by ImageJ software (Fig. 1B). The size values of FePt/GO NSs lays mostly around $500 \mathrm{~nm}$ with some lower value as $100 \mathrm{~nm}$ (Fig. 1C). The existence of some large values $(5000 \mathrm{~nm})$ was due to nanosheet aggregation. The surface atomic composition and oxygen-containing groups were shown in Figure 1D-E. The survey spectra (Fig. 1D) suggested that $\mathrm{C}, \mathrm{O}, \mathrm{Fe}$ and Pt existed on the surface of FePt/GO

NSs and that the relative content of each elements were $40.45 \%, 34.33 \%$, $14.19 \%$ and $11.03 \%$, respectively. In the FRIT spectra, the strong and broad peak around $3400 \mathrm{~cm}^{-1}$ was attribute to the $-\mathrm{OH}$ (Fig. 1E). The peak at 1622 $\mathrm{cm}^{-1}$ resulted from the vibration of undried water molecules, which also contributed to the strong peak at 3400 $\mathrm{cm}^{-1}$. The peaks at $1732 \mathrm{~cm}^{-1}, 1250 \mathrm{~cm}^{-1}$, $1060 \mathrm{~cm}^{-1}$ and $833 \mathrm{~cm}^{-1}$, corresponding to the $\mathrm{C}=\mathrm{O}$, the $\mathrm{C}-\mathrm{OH}$, the $\mathrm{C}-\mathrm{O}-\mathrm{C}$ and the $-\mathrm{CH}(\mathrm{O}) \mathrm{CH}$ (epoxy group), respectively. When the FePt NPs decorated on GO, the oxygen-containing groups were decreased. Meanwhile, the peaks at $1556 \mathrm{~cm}^{-1}$ and $1411 \mathrm{~cm}^{-1}$ belonged to FePt NPs appeared. These results indicated that the FePt NPs had assembled on the surface of GO successfully.

\section{Intracellular distribution of FePt/GO NSs}

The absorption and intercellular localization of $\mathrm{FePt} / \mathrm{GO}$ NSs might play important roles in the biologic effects. The distribution of $\mathrm{FePt} / \mathrm{GO}$ NSs was observed by TEM (Fig. 2). The FePt/GO NSs were internalized either as free or as membrance-boud in cytoplasm. Fig. 2A demonstrated the uptake process, during which the 
FePt/GO NSs entered cells through the endocytic pathway. The FePt NPs were aggregated on the surface of GO, and partly released into cytoplasm with numerous organelles (eg, lysosome, mitochondrion) surrounding (Fig. 2B-C).

\section{FePt/GO NSs inhibited the proliferation of tumor cells without obvious cytotoxicity to normal lung cells.}

To estimate the cytotoxicity of FePt/GO NSs in normal and malignant tumour cell lines, the CCK-8 assay was conducted. Human NSCLC cells (H1975) were incubated with $\mathrm{FePt} / \mathrm{GO}$ NSs over the range of dosages from 0 to $100 \mu \mathrm{g} / \mathrm{mL}$ for $24 \mathrm{~h}$. The cell viability decreased dramatically at the concentration range from 0 to $50 \mu \mathrm{g} / \mathrm{mL}$ (Fig. 3A). The half maximal inhibitory concentration $\left(\mathrm{IC}_{50}\right)$ was found to be 25 $\mu \mathrm{g} / \mathrm{mL}$, which was used in following experiments. The relative cell viability of HeLa, A549, H1299, H460 and HELF cells were examined with different concentration of FePt/GO NSs for $24 \mathrm{~h}$ (Fig. 3B). FePt/GO NSs decreased cell viability in a dose-dependent manner. FePt/GO NSs at $25 \mu \mathrm{g} / \mathrm{mL}$ reduced cell viability on malignant cancer cells by $60-70 \%$ without significant effects on normal lung cells. Although FePt/GO NSs induced a slight decrease of normal cell viability still above the non-cytotoxic threshold of $70 \%$ defined by ISO standard [26].

To investigate the potential mechanisms of $\mathrm{FePt} / \mathrm{GO}$ NSs effect on the cells, ROS generation was measured by the DCFH-DA probe. The ROS levels were significantly upregulated by $\mathrm{FePt} / \mathrm{GO} \mathrm{NS}$ in a dose-dependent manner compared to control group (Fig. 3C-D). The cytotoxicity was accompanied by a corresponding extent of increased intracellular ROS level when exposed to FePt NSs. H1975 cell viability of NAC combined with FePt/GO NSs group was significantly elevated compared with FePt/GO NSs alone (Fig. 3E), indicating that FePt/GO NSs inhibited of lung cancer cell proliferation via up-regulating ROS, which might impair mitochondrial function.

\section{FePt/GO NSs enhanced the radiation sensitivity in $\mathrm{H} 1975$ cell line}

Radiotherapy is a typical therapeutic method for cancer treatment, but the hypoxic and antiradiation of actual tumor reduce the lethality of X-ray. The emergence of radiosensitizer, such as $\mathrm{Au}, \mathrm{Ag}$ and cerium oxide nanoparticles has improved the situation [27-29]. In order to investigate whether $\mathrm{FePt} / \mathrm{GO}$ NSs could increase the radiotherapy sensitivity of H1975, FePt/GO NSs were added into cells pre or postirradiation. Treatment before radiation had a stonger inhibitory effect on cancer cell growth (Fig. 4A) For instance, cell viabilities shown $\sim 5.3 \%, \sim 8.7 \%$, and $\sim 11.4 \%$ differences between the 2 groups at 10, 15, and $25 \mathrm{ug} / \mathrm{mL}$ of the FePt/GO NSs, respectively. The survival cruve which was fitted by "multitarget-single hitting" model indicated FePt/GO NSs enhanced the radiation sensitivity of H1975 cells (Fig. 4B). The results indicated that FePt/GO NSs had synergistic effects with $\mathrm{X}$-ray and might be a potential radiosensitizer. The flow cytometric analysis of annecxin $\mathrm{V}$ and PI indicated that the combination of FePt/GO NSs and radiation led to a slighter increase of apoptosis while individual treatments showed no obvoious apoptosis compared with control (Fig. 4D).

\section{FePt/GO NSs increased autophagy in H1975 cell line}

To investigate whether FePt/GO NSs could increase autophagy, we performed MDC staining, western blotting and TEM analyses after treatments of H1975 cells with FePt/GO NSs and/or X-ray for $24 \mathrm{~h}$. The numbers of MDC-labeled vesicles in H1975 cells were slight increased either exposed to FePt/GO NSs or X-ray beam, and were obvious increased when FePt/GO NSs combined with X-ray beam (Fig. 5A-B). The synergic effect was consistent with cytotoxicity, which implied a relationship between autophagy and cytotoxicity.
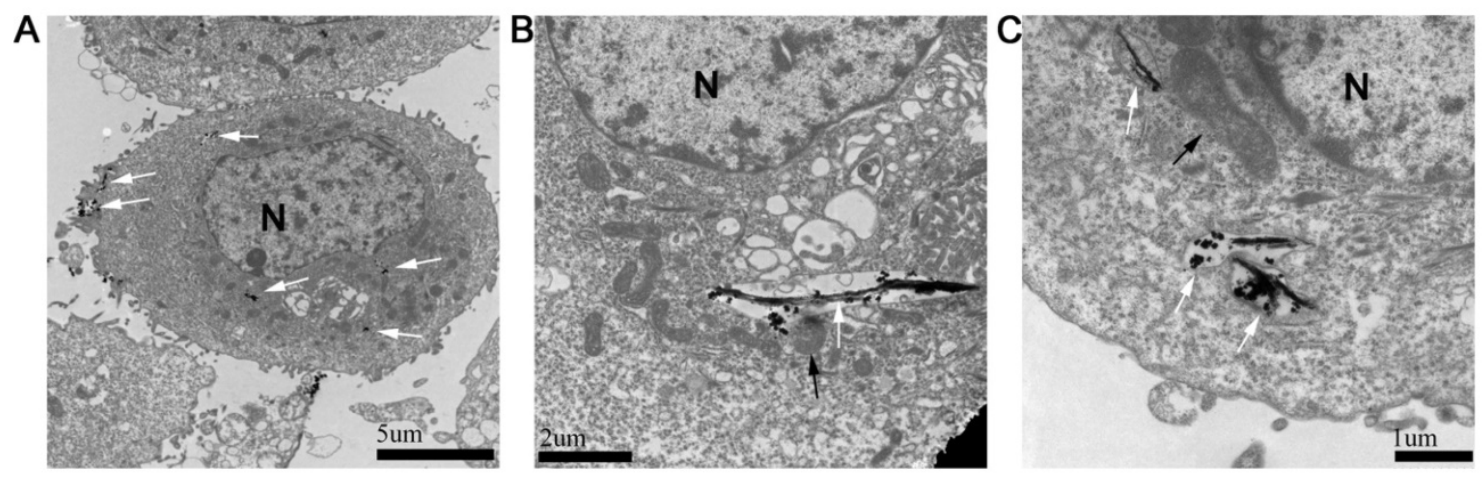

Figure 2. Cellular uptake of FePt/GO NSs by H1975 cells. (A) The distribution of FePt/GO NSs (white arrow) in H1975 cells $24 \mathrm{~h}$ after explosion to 25 $\mathrm{ug} / \mathrm{mL} \mathrm{FePt} / \mathrm{GO}$ NSs. (B) Some FePt NPs partially disembled from GO and attached to surrounding organelles (lysosome, black arrow). (C) Some FePt NPs totally disembled from GO (mitochondria, black arrow). 
A

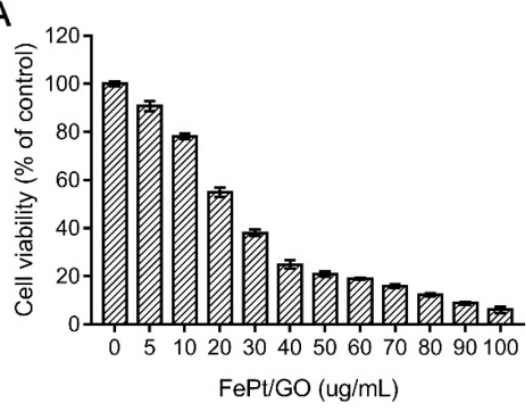

C

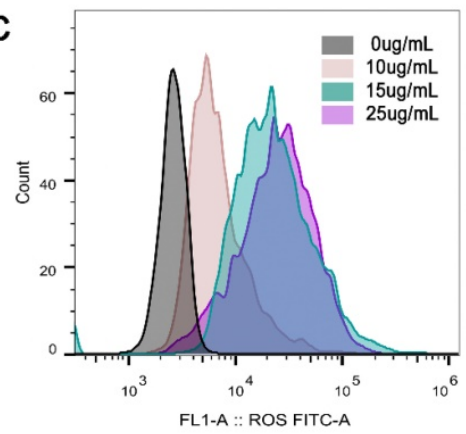

E

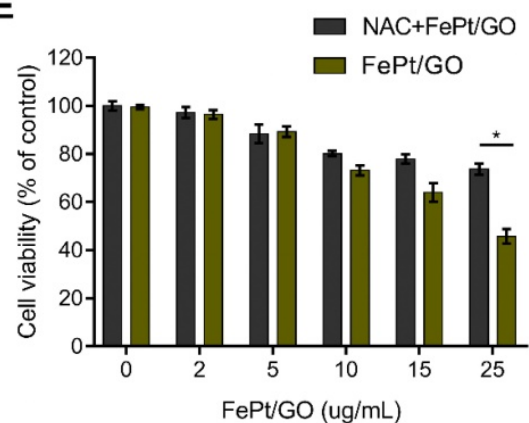

B
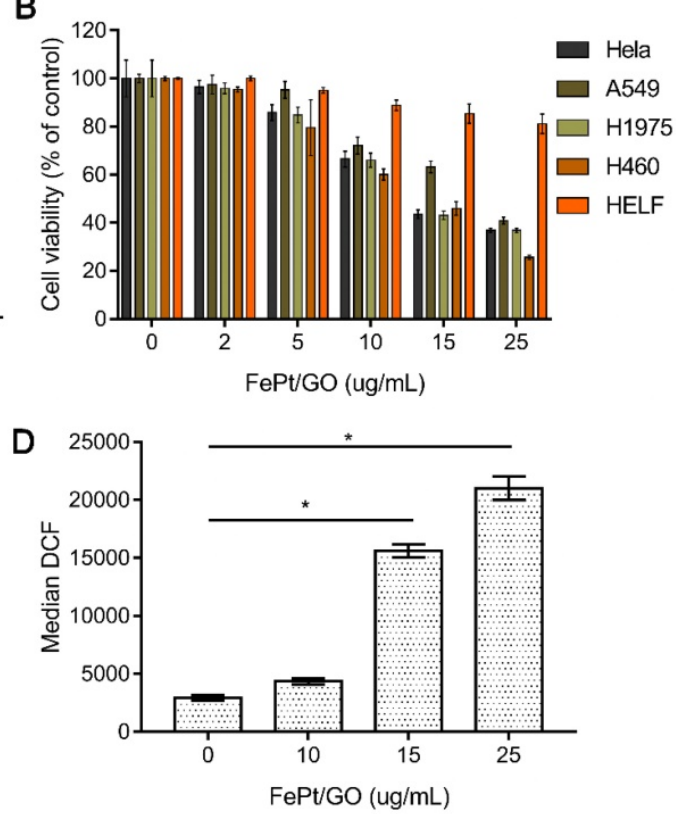

Figure 3. Cytotoxicity of FePt/GO NSs. (A) Cell viability of H1975 cells treated with FePt/GO NS (0-100 ug/mL) for 24 h. IC 50 was 25 ug/mL. (B) Cell viability of H1975, Hela, A549, H460 and HELF cells after treated with FePt/GO NSs (0-25 ug/mL) for 24 h. (C) Cytometry analysis of intercellular ROS levels in H1975 cells after exposed to FePt NSs $(0,10,15,25 \mathrm{ug} / \mathrm{mL})$ for $24 \mathrm{~h}$. (D) The flow cytometric analysis of fluorescence intensity. Data are presented as means \pm SD, *, $<<0.05$. (E) NAC pretreatment significantly decreased the cytotoxicity of FePt/GO NSs in H1975 cells. *, p $<0.05$.

Western blotting analyses (Fig. 5C-D) indicatd increased expression of LC3IIprotein either exposed to FePt/GO NSs or X-ray. The degradation substrate of autophagy p62 was frequently used as marker for autophagy flux [30]. The expression levels of p62 protein were decreased, while those of Beclin1 increased. Both decreased p62 levels and increased Beclin1 levels correlated with enhanced autophagy [31]. To confirm these observations, we performed TEM analyses, which are considered the gold standard for investigations of autophagy in control group and combined therapy group (Fig. 5E). Typical double membrane structure vesicle containing portions of the cytoplasm was found. The late autophagy vacuoles (AVd) contained partially degraded cytoplasmic material. Compared to the control group, FePt/GO NSs combined radiation group showed increased numbers of autophagosomes. These data together demonstrated the formation of autophagosome without autophagy flux blockade.

\section{FePt/GO NSs increased radiation sensitivity in vivo}

LLC cells $\left(6 \times 10^{6}\right.$ cells $)$ were implanted subcutaneously into the left lower limb of C57BL/6 mice. When the tumor volume reached $\sim 100 \mathrm{~mm}^{3}$, treatment was initiated as the scheme showed (Fig. $6 \mathrm{~A})$. The accuracy of radiation filed was evaluated by the X-rays fluoroscopy (Fig. 6B). FePt/GO NSs significantly inhibited tumor growth, and the combination therapy exhibited synergistic effects (Fig. 6C-D). The tumor/body weight was much lower in $\mathrm{FePt} / \mathrm{GO}$ NS group than the control group $(0.041 \pm$ 0.005 vs $0.071 \pm 0.004, p<0.05)$. The combination therapy further decreased then tumor weight compaed with radiation alone $(0.011 \pm 0.003$ vs $0.026 \pm$ $0.004, p<0.05$, Fig. $6 \mathrm{E}$ ). The toxicity of FePt/GO NSs was evaluated by H\&E staining of liver, kidney and heart. No obvious histopathological abnormalities were observed, suggesting $\mathrm{FePt} / \mathrm{GO}$ NSs had no apparent toxicity (Fig. 6F). 
A

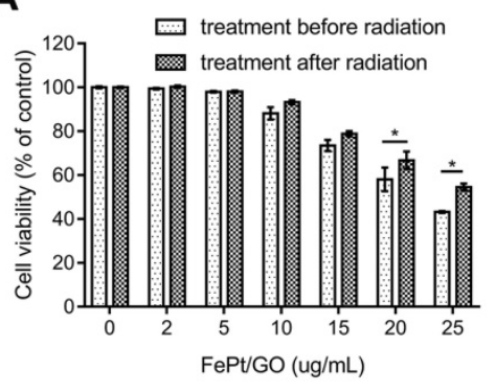

B

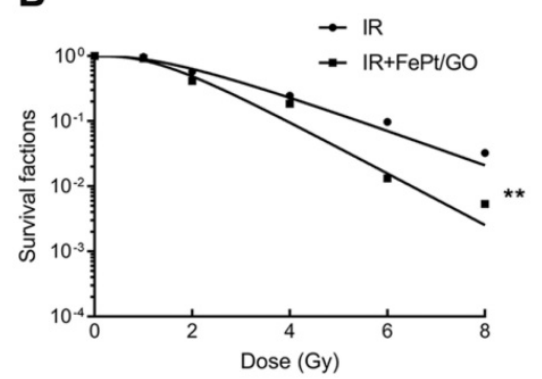

C

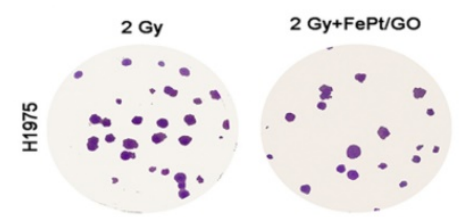

D
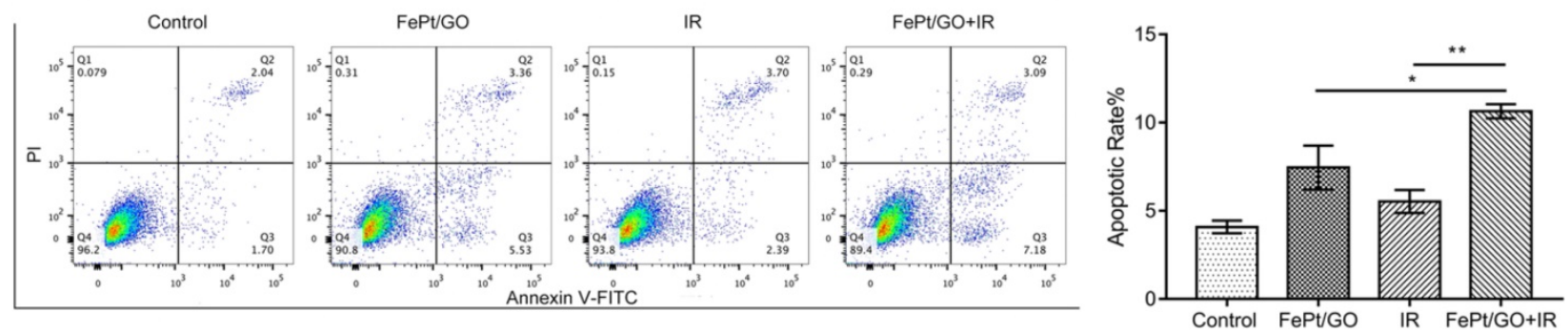

Figure 4. FePt/GO NSs enhanced the radiation sensitivty of $\mathrm{H} 1975$ cells. (A) Cell viability of $\mathrm{H} 1975$ cells treated with FePt/GO NSs (25 ug/mL) before or after radiation for $24 \mathrm{~h}$. *, p $<0.05$. (B) The survival fraction of FePt/GO NSs $(25 \mathrm{ug} / \mathrm{mL}$ ) were significant lower than that of vehicle control. (C) Representative crystal violet stainning of $\mathrm{H} 1975$ cells irradiated with $2 \mathrm{~Gy}$ with or without $\mathrm{FePt} / \mathrm{GO}$ NS treatment $(25 \mathrm{ug} / \mathrm{mL}$ ). (D) Representative cytometry analysis of annexin $\mathrm{V}$ and PI in $\mathrm{H} 1975$ cells. The double treatment group had more cell apoptosis than individual ones. Statistical analysis reveals the apoptotic rate (\%) of $\mathrm{H} 1975$ cells after different treatment, $*, p<0.05, * *, p<0.01$.

\section{Discussion}

To date, the development of nanomaterials has improved the diagnosis and treatment of cancers. FePt nanocomposites showed the potential applications in dual modal MRI/CT imaging [5, 6], drug delivery [7], thermal therapy [8,9] and chemotherapy agents [32] owing to their optimal magnetic, optical properties and controllable morphology. Chen et al. [33] synthesized FePt/GO nanocomposites with good activity of intrinsic peroxidase. Yue et al. [5] developed a magnetic nanocomposites named FePt-DMSA/GO-PEG-FA (FePt/GO CNs). FePt/GO $\mathrm{CNs}$ has potential for multimodal imaging and inhibition of 4T1 tumor growth. In the present work, we successfully synthesized FePt/GO nanocomposites and used various characterization techniques to clarify surface morphology, chemical composition and surface oxide groups. Compared with $\mathrm{FePt}$ nanocomposites, FePt/GO NSs had flexible size, abundant oxygen-containing groups and excellent biocompatibility. As shown in Fig. 2A, H1975 cells uptaked FePt NSs through endocytosis for cell membrane invaginations. Most internalized nanoparticles by endocytic routes are principally aggregated in the lysosome. Once FePt NSs entered into cytoplasm, FePt NPs detached from the surface of reduced GO and deposited in mitochondria or lysosome (Fig. 2B-C).

To assess whether FePt/GO NSs could serve as an agent in chemoradiotherapy, a series of toxicity and sensitization experiments were conducted in vitro. Firstly, FePt/GO NSs showed a dose-dependent cytotoxicity in H1975 cells (Fig. 3A). Secondly, FePt/GO NSs exhibited significantly cytotoxicity to human lung cancer cells (A549, H1975 and H460) and cervic cancer cells (HeLa), but not obvious cytotoxicity to the normal lung cells (HELF). In other words, $\mathrm{FePt} / \mathrm{GO}$ NSs selectively inhibited the proliferation of cancer cells. Due to intrinsic properties, FePt NPs was reported to release highly active $\mathrm{Fe}$ ions during the degradation in lysosome and catalyse $\mathrm{H}_{2} \mathrm{O}_{2}$ decomposition into ROS, leading to oxidative stress [11]. We next measured intracellular ROS production in H1975 cells treated with different concentrations of FePt/GO NSs. The ROS levels were significantly upregulated by FePt/GO NSs in a dose-dependent manner compared to control group (Fig. 3C-D). The ROS scavenger NAC had significant elevate cell survival rate compared to corresponding FePt/GO NSs treatment alone (Fig. 3E). Excessive ROS leaded to oxidative damage of proteins, lipids and DNA, and finally induced apoptosis or growth inhibit [34]. Taken together, the cytotoxicity induced by FePt/GO NSs might be through ROS. Moreover, cancer cells were reported to have a higher basal ROS levels than healthy cells [35], which suggested cancer cells could be vulnerable group under oxidative stress. In addition, similar selective cytotoxicity was reported in other nanoparticles [5, 20, 36, 37]. For instance, bare iron oxide NPs induced ROS increasement and 
mitochondrial damage in lung cancer cells (A549), without significant toxicity to normal human lung fibroblast cells (IMR-90) [21]. Zinc oxide NPs selectively induced apoptosis in HepG2 cells which was mediated by ROS via p53 pathway [37]. These results suggested taht the selective cytotoxicity might be mediated by production of ROS induced by NPs.

We further explored whether FePt/GO NSs could sensitilize H1975 cells combined with X-rays. The synergetic group (treatment before radiation) showed greater proliferation suppression ratios compared with the simple superposition group (treatment after radiation) (Fig. 4A). Furthermore, the survival cruve (fitted by "multitarget-single hitting" model) also confirmed that FePt/GO NSs enhanced the radiation sensitivity of $\mathrm{H} 1975$ cells (Fig. 4B). However the results of cell cycle assay showed no significant difference between control group and FePt/GO NSs group (data not showed). Meanwhile, no obvious G1 or G2 phase arrest were observed between IR group and combined group. Moreover, FePt/GO NSs combined IR triggered apoptosis pathway in a moderate way, while FePt/GO NSs group or IR group alone showed no elevated apoptotic rate (Fig. 4D).
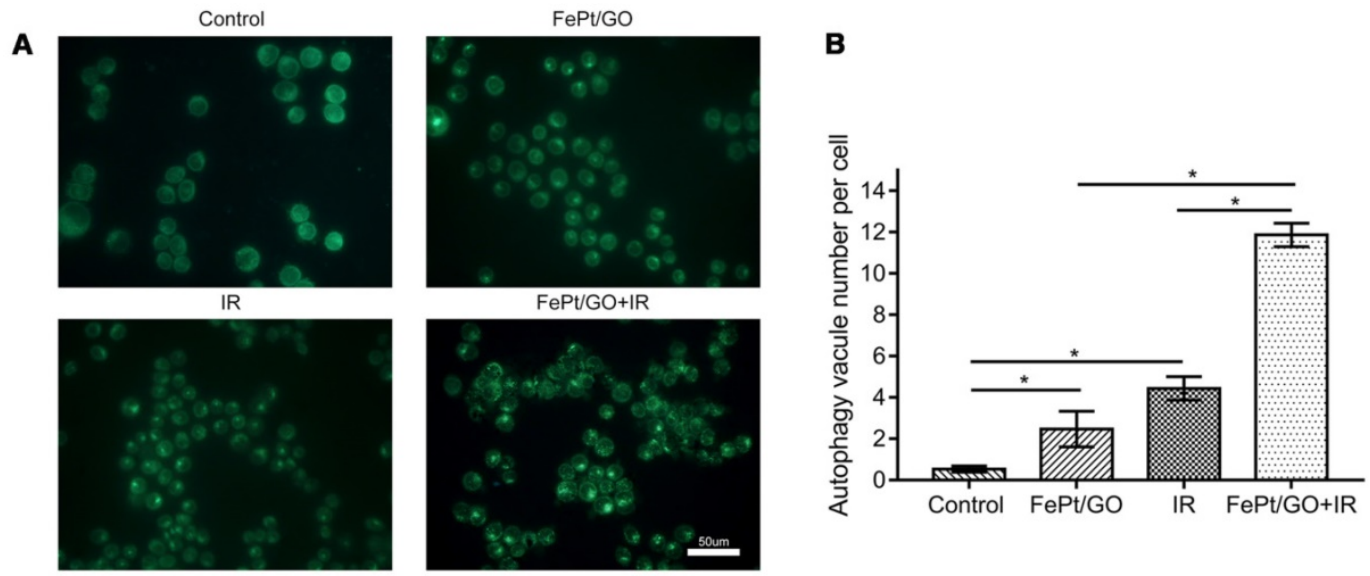

C

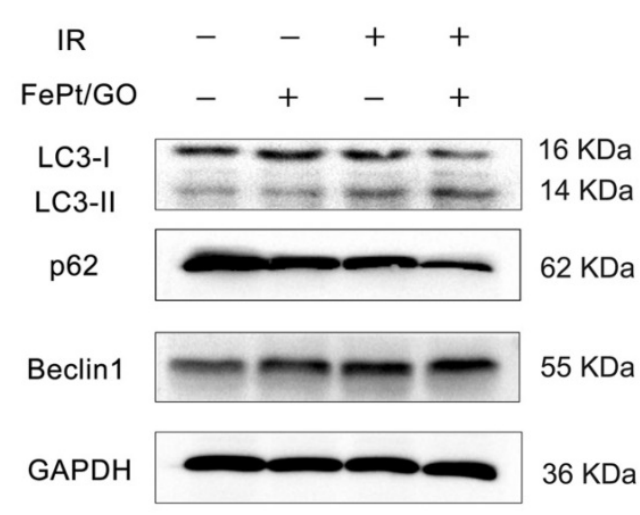

。

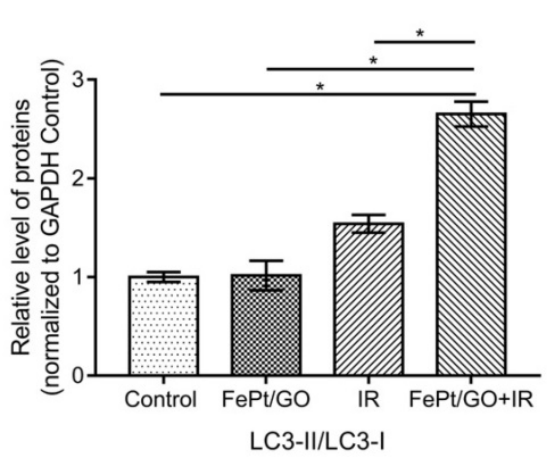

$\mathbf{E}$

\section{Control}

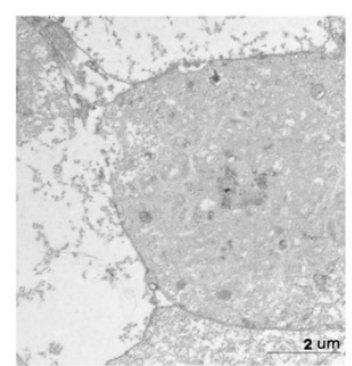

FePt/GO

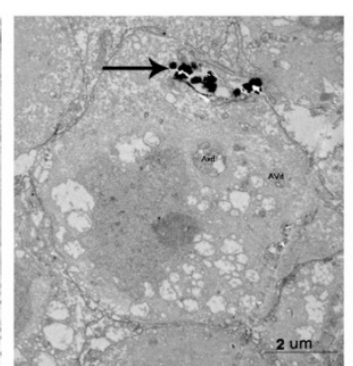

IR

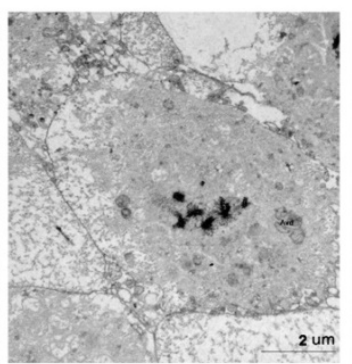

FePt/GO+IR

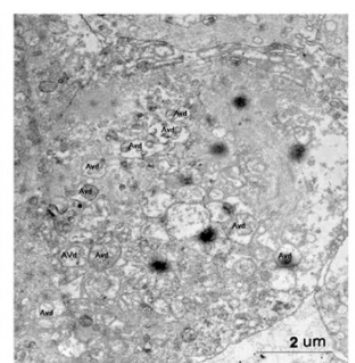

Figure 5. FePt/GO NSs promoted autophagy in H1975 cells induced by X-ray. (A) Representative fluorescent images of MDC staining in irradiated (4 Gy) H1975 cells treated with or without FePt/GO NSs (25 ug/mL). scale bar: 50 um. (B) Statistical analysis of green fluorescent spot number. All values shown are means \pm SD of triplicate tests and are repeated three times with similar results. (C) Representative western blotting of LC3, p62 and Beclin1 in H1975 cells. (D) Relative expression levels of the ratio LC3-II/LC3-I(Grey value) from 3 replicate experiments are quantitated and subjected to statistical analysis. (E) Representative TEM images for autophagosomes in four groups, scale bar: 2 um. (AVd: the late autophagy vacuoles; black arrows: FePt/GO nanosheets). 
A

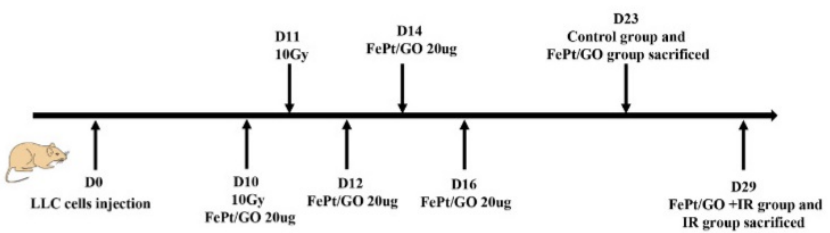

B

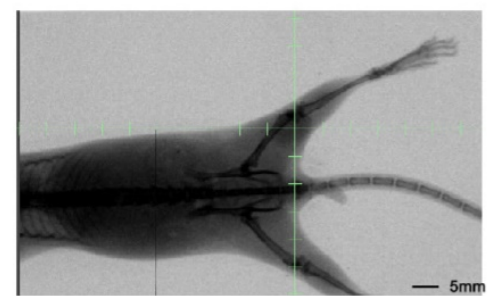

C

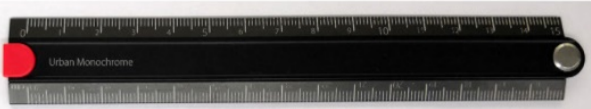

Control

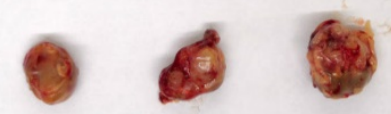

FePt/GO

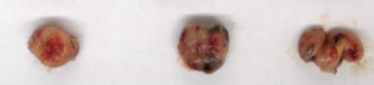

IR

FePt/GO+IR

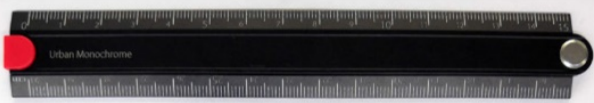

$\mathbf{F}$

Control

FePt/GO
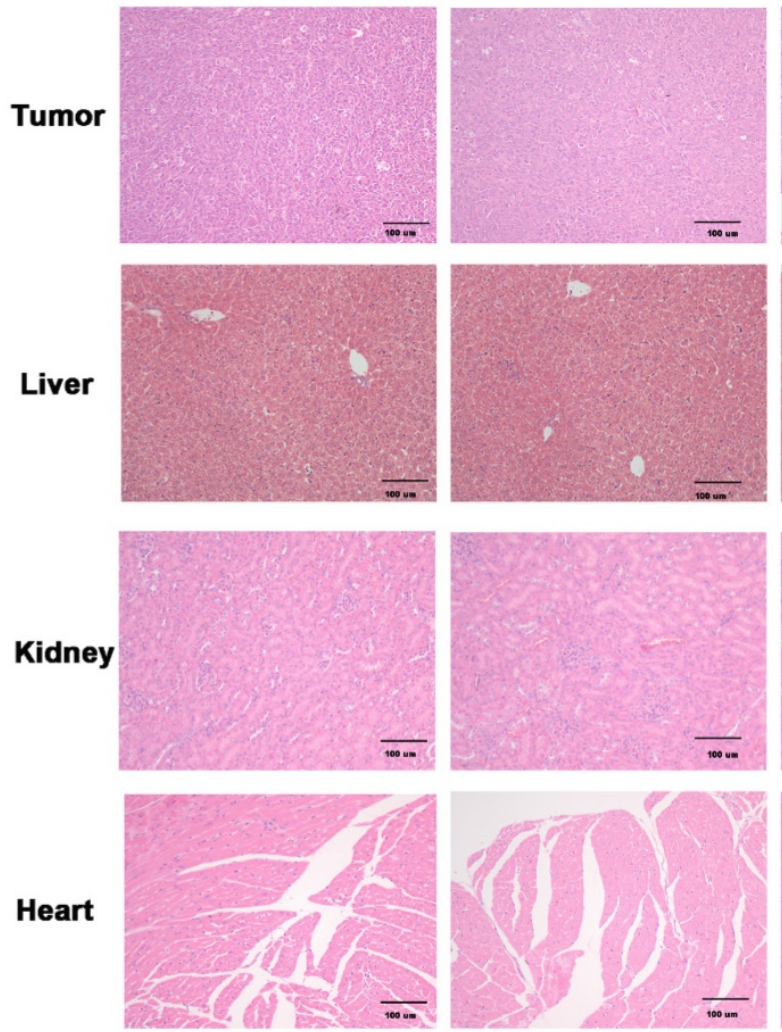

D

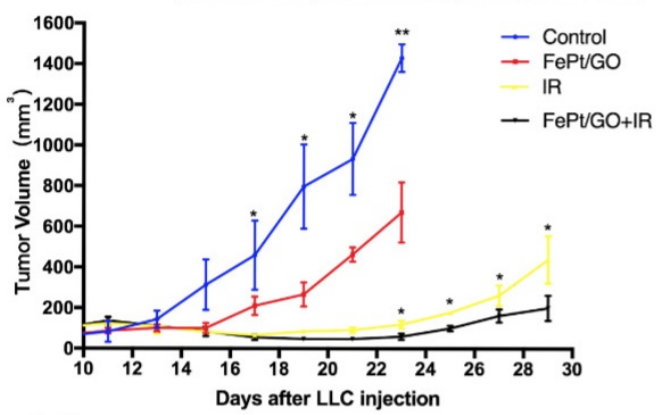

E

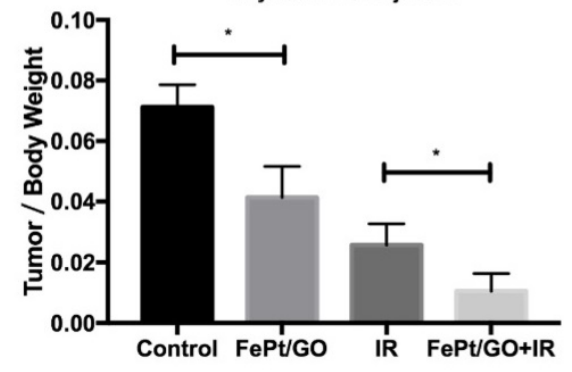

IR

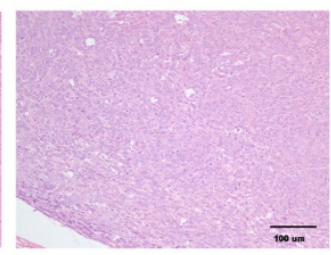

FePt/GO+IR
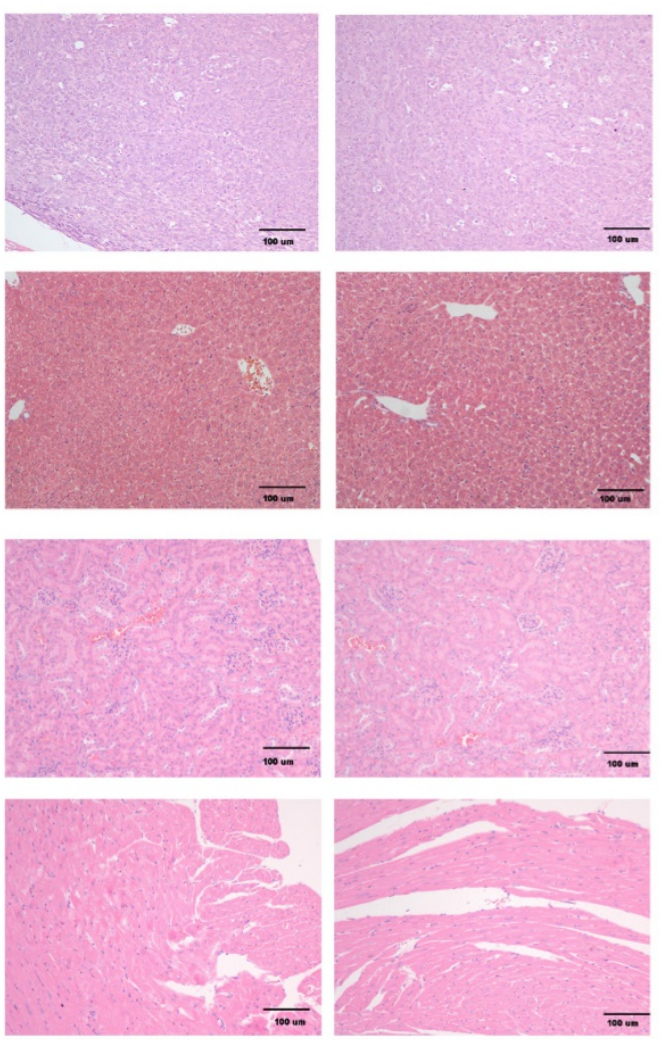

Figure 6. FePt/GO NSs increased radiation sensitivity in vivo. (A) Treatment scheme of animal study. (B) Representative image of $X$-rays fluoroscopy before irradiation, scale bar: $5 \mathrm{~mm}$. (C) Gross view of tumor. (D) Growth curve of tumor volume. $N=3 ; *, p<0.05$. (E) Tumor/Body weight ratio. $N=3$; *, $P<0.05$. (F) Representative panels of HE staining of tumor, liver, kidney and heart of mice, scale bar: $5 \mathrm{~mm}$. 
Accumulated data suggested an essential role of ROS in the activation of autophagy [38]. Autophagy is a homeostatic and evolutionarily conserved intracellular process for celluar degradation of redundant or faulty cell components [39]. It was reported that induction of ROS and increased level of radiationinduced autophagy was responsible for radioresistance in pancreatic cancer [40]. So we assumed potential relation between autophagy and FePt/GO NSs. MDC staining was widely reported to label autophagic vacuoles in vitro. Green fluorescence increased in FePt/GO NSs or IR group alone, while combined group showed remarkable elevation (Fig. 5A-B). The formation of autophagosome involves complex multistep process which is coordinated by some key proteins, such as Beclin1 and LC3. At the beginning of autophagy, Beclin1 acts as a platform to recruit other proteins which are needed for autophagosome formation. Then, LC3-I transforms into LC3-II by conjugating with lipid phosphatidylethanolamine and subsequently incorporates into the double membranes of autophagosome. The increase of Beclin1 and LC3-II (Fig. 5C) indicated the formation of autophagosome. The protein p62 interacts directly to LC3 and forms proteins aggregates which is selectively degraded by autophagy [41]. SiNPs was reported to upregulate p62 by inhibiting the degradation of autophagosome [42]. Our results showed smooth autophagy flux without any blockage, which was consistent with TEM observation (Fig. 5E). The vivo study also suggested FePt/GO NSs exerted synergistic effects with radiation without obvious toxicity (Fig. 6D-E).

\section{Conclusions}

In this study, we reported the synthesis and characterization of FePt/GO NSs. With good safety, it selectively inhibited the proliferation of NSCLC in vitro and in vivo, increased the ROS production in a dose dependant manner, improved the radiosensitivity of lung cancer cells, and induced apoptosis when combined with X-ray. Meanwhile, we detected autophagy induced by FePt/GO NSs, which might contribute to its radiosensitizing effect.

\section{Abbreviations}

NSCLC: Non-small cell lung cancer; FePt/GO NSs: FePt/GO nanosheets; GO: graphene oxide; TEM: transmission electron microscope; DLS: dynamic light scattering; XPS: X-ray photoelectron spectroscope; FTIR: Fourier transform infrared spectroscopy; ROS: reactive oxygen species; NAC: N-acetylcysteine; MDC: monodansylcadaverine; PBS: phosphate buffered saline; DCFH-DA: 2, 7-dichlorodihydrofluorescein diacetate; CCK-8: Cell Counting Kit-8.

\section{Acknowledgements}

The excellent technical assistance of Ms. Xiaohua Leng is gratefully acknowledged.

\section{Grant support}

This study was supported in part by grants from the Chinese National Natural Science Foundation (grant No. 81572967, 81800429, and 81773236), Hubei Natural Science Foundation (grant No. 2013CF A006), Zhongnan Hospital of Wuhan University Science, Technology and Innovation Seed Fund (grant No. znpy2016050, znpy2017001, and znpy2017049), National key clinical speciality construction program of China (No. [2013]544), Wuhan City Huanghe Talents Plan, and the Fundamental Research Funds for the Central Universities (grant No. 2042018kf0065).

\section{Author Contributions}

Conceived and designed the study: S.M., H.M., H.Q. and C.X. Performed the study: S.M., H.M., Y.L., Y.S., X.T., F.W., C.Y., and S.P. Analyzed the results: S.M., H.M., G.T., C.Y., Y.G., H.Q., and C.X. Contributed reagents/ materials/analysis tools: W.S., S.L., Y.M., J.X., and Y.X. Wrote the manuscript: S.M., H.M., Y.G., H.Q., and C.X. All authors reviewed and agreed to the publication of the manuscript.

\section{Competing Interests}

The authors have declared that no competing interest exists.

\section{References}

1. Torre LA, Bray F, Siegel RL, Ferlay J, Lortet-Tieulent J, Jemal A. Global cancer statistics, 2012. CA Cancer J Clin. 2015; 65: 87-108.

2. Torre LA, Siegel RL, Jemal A. Lung Cancer Statistics. In: Ahmad A, Gadgeel S, editors. Lung Cancer and Personalized Medicine: Current Knowledge and Therapies. Cham: Springer International Publishing; 2016. p. 1-19.

3. Zatloukal P, Petruzelka L, Zemanova M, Havel L, Janku F, Judas L, et al. Concurrent versus sequential chemoradiotherapy with cisplatin and vinorelbine in locally advanced non-small cell lung cancer: a randomized study. Lung Cancer. 2004; 46: 87-98.

4. Curran Jr WJ, Paulus R, Langer CJ, Komaki R, Lee JS, Hauser S, et al. Sequential vs concurrent chemoradiation for stage III non-small cell lung cancer: randomized Phase III trial RTOG 9410. Journal of the National Cancer Institute. 2011; 103: 1452-60.

5. Yue L, Wang J, Dai Z, Hu Z, Chen X, Qi Y, et al. pH-Responsive, Self-Sacrificial Nanotheranostic Agent for Potential In Vivo and In Vitro Dual Modal MRI/CT Imaging, Real-Time, and In Situ Monitoring of Cancer Therapy. Bioconjug Chem. 2017; 28: 400-9.

6. Chou S-W, Shau Y-H, Wu P-C, Yang Y-S, Shieh D-B, Chen C-C. In vitro and in vivo studies of FePt nanoparticles for dual modal CT/MRI molecular imaging. Journal of the American Chemical Society. 2010; 132: 13270-8.

7. Fuchigami T, Kawamura R, Kitamoto Y, Nakagawa M, Namiki Y. A magnetically guided anti-cancer drug delivery system using porous FePt capsules. Biomaterials. 2012; 33: 1682-7.

8. Chen CL, Kuo LR, Lee SY, Hwu YK, Chou SW, Chen CC, et al. Photothermal cancer therapy via femtosecond-laser-excited FePt nanoparticles. Biomaterials. 2013; 34: 1128-34.

9. Sahu NK, Gupta J, Bahadur D. PEGylated FePt-Fe3O4 composite nanoassemblies (CNAs): in vitro hyperthermia, drug delivery and generation of reactive oxygen species (ROS). Dalton Trans. 2015; 44: 9103-13.

10. Sun Y, Miao H, Ma S, Zhang L, You C, Tang F, et al. FePt-Cys nanoparticles induce ROS-dependent cell toxicity, and enhance chemo-radiation sensitivity of NSCLC cells in vivo and in vitro. Cancer Lett. 2018; 418: 27-40.

11. Xu C, Yuan Z, Kohler N, Kim J, Chung MA, Sun S. FePt nanoparticles as an Fe reservoir for controlled Fe release and tumor inhibition. J Am Chem Soc. 2009; 131: 15346-51. 
12. Zheng Y, Tang Y, Bao Z, Wang H, Ren F, Guo M, et al. FePt nanoparticles as a potential X-ray activated chemotherapy agent for HeLa cells. Int J Nanomedicine. 2015; 10: 6435-44.

13. Klionsky DJ. Autophagy: from phenomenology to molecular understanding in less than a decade. Nature reviews Molecular cell biology. 2007; 8: 931-7.

14. Yang ZJ, Chee CE, Huang S, Sinicrope FA. The role of autophagy in cancer: therapeutic implications. Mol Cancer Ther. 2011; 10: 1533-41.

15. Kondo Y, Kanzawa T, Sawaya R, Kondo S. The role of autophagy in cancer development and response to therapy. Nat Rev Cancer. 2005; 5: 726-34.

16. Stern ST, Adiseshaiah PP, Crist RM. Autophagy and lysosomal dysfunction as emerging mechanisms of nanomaterial toxicity. Particle and fibre toxicology. 2012; 9: 20.

17. Ma X, Wu Y, Jin S, Tian Y, Zhang X, Zhao Y, et al. Gold nanoparticles induce autophagosome accumulation through size-dependent nanoparticle uptake and lysosome impairment. ACS nano. 2011; 5: 8629-39.

18. Chiu H-W, Ho S-Y, Guo H-R, Wang Y-J. Combination treatment with arsenic trioxide and irradiation enhances autophagic effects in U118-MG cells through increased mitotic arrest and regulation of PI3K/Akt and ERK1/2 signaling pathways. Autophagy. 2014; 5: 472-83

19. Halamoda Kenzaoui B, Chapuis Bernasconi C, Guney-Ayra S, Juillerat-Jeanneret L. Induction of oxidative stress, lysosome activation and autophagy by nanoparticles in human brain-derived endothelial cells. Biochem J. 2012; 441: 813-21.

20. Khan MI, Mohammad A, Patil G, Naqvi SA, Chauhan LK, Ahmad I. Induction of ROS, mitochondrial damage and autophagy in lung epithelial cancer cells by iron oxide nanoparticles. Biomaterials. 2012; 33: 1477-88.

21. Yu Y, Duan J, Yu Y, Li Y, Liu X, Zhou X, et al. Silica nanoparticles induce autophagy and autophagic cell death in HepG2 cells triggered by reactive oxygen species. J Hazard Mater. 2014; 270: 176-86.

22. Lopes VR, Loitto V, Audinot JN, Bayat N, Gutleb AC, Cristobal S. Dose-dependent autophagic effect of titanium dioxide nanoparticles in human HaCaT cells at non-cytotoxic levels. J Nanobiotechnology. 2016; 14: 22.

23. Chen Y, Azad MB, Gibson SB. Superoxide is the major reactive oxygen species regulating autophagy. Cell Death Differ. 2009; 16: 1040-52.

24. Hummers Jr WS, Offeman RE. Preparation of graphitic oxide. Journal of the American Chemical Society. 1958; 80: 1339-.

25. Eric J. Hall AJG. Radiobiology for the Radiologist: LWW; 2018.

26. ISO I. 10993-5: 2009 Biological Evaluation of Medical Devices-Part 5: Tests for in Vitro Cytotoxicity. International Organization for Standardization, Geneva. 2009.

27. Geng F, Song K, Xing JZ, Yuan C, Yan S, Yang Q, et al. Thio-glucose bound gold nanoparticles enhance radio-cytotoxic targeting of ovarian cancer. Nanotechnology. 2011; 22: 285101

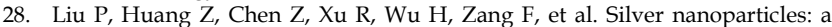
novel radiation sensitizer for glioma? Nanoscale. 2013; 5: 11829-36.

29. Wason MS, Colon J, Das S, Seal S, Turkson J, Zhao J, et al. Sensitization of pancreatic cancer cells to radiation by cerium oxide nanoparticle-induced ROS production. Nanomedicine: Nanotechnology, Biology and Medicine. 2013; 9: $558-69$

30. Bjørkøy G, Lamark T, Pankiv S, Øvervatn A, Brech A, Johansen T. Chapter 12 Monitoring Autophagic Degradation of p62/SQSTM1. Methods in Enzymology: Academic Press; 2009. p. 181-97.

31. Kara N, Toker L, Agam G, Anderson G, Belmaker R, Einat H. Trehalose induced antidepressant-like effects and autophagy enhancement in mice. Psychopharmacology. 2013; 229: 367-75.

32. Bao Z, He M, Quan H, Jiang D, Zheng Y, Qin W, et al. FePt nanoparticles: a novel nanoprobe for enhanced HeLa cells sensitivity to chemoradiotherapy. RSC Adv. 2016; 6: 35124-34.

33. Chen $M$, Yang $B$, Zhu I, Liu $H$, Zhang $X$, Zheng $X$, et al. FePt nanoparticles-decorated graphene oxide nanosheets as enhanced peroxidase mimics for sensitive response to H2O2. Mater Sci Eng C Mater Biol Appl. 2018; 90: 610-20.

34. Martindale JL, Holbrook NJ. Cellular response to oxidative stress: signaling for suicide and survival. Journal of cellular physiology. 2002; 192: 1-15.

35. Schumacker PT. Reactive oxygen species in cancer cells: Live by the sword, die by the sword. Cancer Cell. 2006; 10: 175-6.

36. Gao W, Ji L, Li L, Cui G, Xu K, Li P, et al. Bifunctional combined Au-Fe2O3 nanoparticles for induction of cancer cell-specific apoptosis and real-time imaging. Biomaterials. 2012; 33: 3710-8.

37. Akhtar MJ, Ahamed M, Kumar S, Khan MM, Ahmad J, Alrokayan SA. Zinc oxide nanoparticles selectively induce apoptosis in human cancer cells through reactive oxygen species. International journal of nanomedicine. 2012; 7: 845 .

38. Scherz-Shouval R, Elazar Z. Regulation of autophagy by ROS: physiology and pathology. Trends Biochem Sci. 2011; 36: 30-8.

39. Guo JY, Xia B, White E. Autophagy-mediated tumor promotion. Cell. 2013; 155: 1216-9.

40. Wang F, Xia X, Yang C, Shen J, Mai J, Kim HC, et al. SMAD4 Gene Mutation Renders Pancreatic Cancer Resistance to Radiotherapy through Promotion of Autophagy. Clin Cancer Res. 2018; 24: 3176-85.

41. Pankiv S, Clausen TH, Lamark T, Brech A, Bruun J-A, Outzen H, et al. p62/SQSTM1 binds directly to Atg8/LC3 to facilitate degradation of ubiquitinated protein aggregates by autophagy. Journal of Biological Chemistry. 2007; 282: 24131-45.
42. Wang J, Yu Y, Lu K, Yang M, Li Y, Zhou X, et al. Silica nanoparticles induce autophagy dysfunction via lysosomal impairment and inhibition of autophagosome degradation in hepatocytes. Int J Nanomedicine. 2017; 12: 809-25. 